\title{
Stochastic analysis of immiscible displacement in porous media
}

\author{
Hwa-Sheng Gau ${ }^{\mathrm{a}}$, Chen-Wuing Liu ${ }^{\mathrm{a}, *} \&$ Yii-Soong Tsao ${ }^{\mathrm{b}}$ \\ ${ }^{a}$ Department of Agricultural Engineering, National Taiwan University, Taipei, Taiwan, Republic of China \\ ${ }^{b}$ Department of Civil Engineering, National I-Lan Institute of Agriculture and Technology, I-Lan, Taiwan, Republic of China
}

(Accepted 26 March 1997)

\begin{abstract}
The interface of two immiscible fluids flowing in porous media may behave in an unstable fashion. This instability is governed by the pore distribution, differential viscosity and interface tension between the two immiscible fluids. This study investigates the factors that control the interface instability at the wetting front. The development of the flow equation is based on the mass balance principle, with boundary conditions such as the velocity continuity and capillary pressure balance at the interface. By assuming that the two-phase fluids in porous media are saturated, a covariance function of the wetting front position is derived by stochastic theory. According to those results, the unstable interface between two immiscible fluids is governed by the fluid velocity and properties such as viscosity and density. The fluid properties that affect the interface instability are expressed as dimensionless parameters, mobility ratio, capillary number and Bond number. If the fluid flow is driven by gravitational force, whether the interface undergoes upward displacement or downward displacement, the variance of the unstable interface decreases with an increasing mobility ratio, increases with increasing capillary number, and decreases with increasing Bond number. For a circumstance in which fluid flow is horizontal, our results demonstrate that the capillary number does not influence the generation of the unstable interface. (C) 1998 Elsevier Science Limited. All rights reserved
\end{abstract}

Key words: instability, wetting front, immiscible fluid, stochastic theory.

\section{INTRODUCTION}

The interface of two immiscible fluids flowing in porous media may behave in an unstable fashion. The instability is caused by pore distribution, differential viscosity, and interface tension between the two immiscible fluids ${ }^{18}$. The unstable phenomenon is called fingering, when the shape of the unstable wetting front resembles fingers. The velocity at the fingertips may be higher than the mean velocity, which may cause the aquifer contaminant to move much faster, subsequently increasing the risk of contaminating groundwater aquifers. Therefore the factors controlling an unstable wetting front must be investigate to prevent and remediate groundwater contamination.

The physical mechanisms controlling the unstable interface between immiscible fluids have long been known to petroleum engineers. Recently, this problem has been reconsidered in the fields of soil physics and subsurface hydrology. Hill ${ }^{14}$ derived a critical velocity, beyond which a finger develops from a perturbation. Chuoke et al. ${ }^{5}$ *Fax: +886-2-3639557; e-mail: liu@gwater.agec.ntu.edu.tw extended the work of Hill $^{14}$ by performing first-order stability analysis on an initial planar interface between two immiscible fluids moving through homogeneous porous media. They not only decomposed the perturbed macroscopic interface into fundamental Fourier perturbation modes, but also studied each of these modes separately. The same critical velocity required for fingering onset as that of Hill ${ }^{14}$ was derived, but with the added constraint that the perturbation contains wavelengths exceeding a critical wavelength. Chuoke $e t$ al. ${ }^{5}$ indicated that both larger viscosity differences and higher velocities lead to smaller finger spacing, and larger interface tensions lead to wider finger spacing. These relationships derived by Chuoke $e t a l^{5}$ have been verified by laboratory studies ${ }^{5}$. Raats ${ }^{29}$ presented a stability criterion for water infiltrating through the unsaturated zone based on the hydraulic model of Green and $\mathrm{Ampt}^{13}$. He showed that a wetting front displacing air at constant atmospheric pressure is inherently stable due to imbibition at the advancing front. Hill and Parlange ${ }^{15}$ studied the unstable condition of two immiscible fluids flowing through two-layer, porous media, with fine soil on the upper 
layer and coarser soil on the lower layer. The unstable wetting front occurred when the flow rate through the coarser layer was less than the saturated hydraulic conductivity. Philip $^{27,28}$ extended the work of Raats ${ }^{29}$ to incorporate the effects of surface tension. He concluded that a wetting front would be unstable if there exists a pressure gradient opposing the direction of flow, and stable if the pressure gradient assists the flow. Based on the assumption that the speed of a perturbed front is affected by its curvature, Parlange and Hill $^{26}$ found that a wetting front moving in a homogeneous soil at a sufficiently low speed is unstable. The wavelength for fine-textured soils is larger than that for coarse-textured soils and increases with an increasing initial moisture content. Mclean and Saffman ${ }^{25}$ extended the work of Saffman and Taylor ${ }^{30}$ to include the effect of surface tension due to interface curvature on a wetting front. Chang ${ }^{4}$ introduced the fractal theory for analysing the wetting front instability in unsaturated soil. According to their results, the fractal dimension can be used as a criterion to predict whether fingering would occur in porous media and the minimum spatial distance required to detect the dominant fingering location. Glass $^{12}$ derived expressions by dimensional analysis for finger width and finger velocity that are functions of porous media properties and initial or boundary conditions. Glass $e t$ al. ${ }^{11}$ extended the analyses of Parlange and $\mathrm{Hill}^{26}$ to investigate finger diameter, including the relationships between the velocity of curves and radii of curvature with axisymmetric disturbances in a three-dimensional system.

Natural porous media, however, contain a distribution of pore sizes that cause the intrinsic permeability to vary in space. Consequently, different parts of an initially planar interface move with different velocities through the porous media, and the wetting front becomes unstable ${ }^{2}$. Within each soil type or geologic unit, the properties of these elements are assumed to originate from frequency distribution. If the distribution is monomodal, the medium is homogeneous; if it is multimodal, it is heterogeneous ${ }^{9}$. From this classification, the outcome of intrinsic permeability is a stochastic process. Consequently, determining the velocity of different parts of the interface between immiscible fluids becomes a stochastic process. Many researchers have investigated the variance of permeability in saturated porous media $a^{1,6,10,31}$ and unsaturated porous media ${ }^{17,22-24,32,33,35}$ by stochastic theory. In this paper, we adopt stochastic theory to derive the autocovariance function of the wetting front position. Parameters that control the interface instability of the wetting front are investigated from the auto-covariance function of wetting front position. In addition, quantitative results are discussed in detail to further understand instability in wetting fronts between immiscible fluids.

\section{INTERFACE CONDITIONS}

\subsection{Capillary pressure and unstable wetting front}

The pore diameter affects the flow path in a porous media. The curvature of the wetting front through larger pores is less than that through finer pores. Therefore, the fluid interface moves easily through media with large pores because the energy stored in the interface quickly exceeds the interface tension. The pore diameter in the actual porous media, however, is a function of position. Thus, the displacement varies in different parts of the interface.

If the interface between the two immiscible fluids is assumed to be an impermeable membrane, the displacement of the interface, which is attributed to the forces around the membrane, will be unbalanced. The differential force of the interface can be represented as follows:

$$
\Delta P=P_{1}-P_{2}
$$

where $P_{1}$ and $P_{2}$ denote the pressures of the two immiscible fluids, and $\Delta P$ represents the capillary pressure between the two immiscible fluids. If the differential displacement $\mathrm{d} h$ is caused by the $\Delta P$, then the work of the interface surface can be expressed as the product of the pressure difference and differential displacement. In addition, the work can be expressed as surface tension work on the surface. Therefore, based on the equality of work ${ }^{4}$, this can be represented as

$$
\left(P_{1}-P_{2}\right) \mathrm{d} h \int \mathrm{d} A_{1}=\sigma \int \mathrm{d}(\Delta A)
$$

where $\mathrm{d} A_{1}$ is a finite surface area, $\sigma\left(M T^{-2}\right)$ is the interface tension, and $\mathrm{d}\left(\Delta A_{1}\right)$ is the net increase in finite surface area as the curvature of the surface is increased. The integral variables are different in eqn (2). Therefore, the relationship between $\mathrm{d} A_{1}$ and $\mathrm{d}\left(\Delta A_{1}\right)$ needs to be established.

Chang ${ }^{4}$ has derived the work for a two-dimension case; it also can be expanded to a three-dimensional case. Let $R_{1 X}$ and $R_{1 Y}$ be the principal radii, $S_{1 X}$ and $S_{1 Y}$ be the length of the principal section curvature, and $\theta_{1 X}$ and $\theta_{1 Y}$ be the angles of the finite principle radii, as shown in Fig. 1. After a small displacement, $\mathrm{d} h$, the principal radii, the length of the principal section curvature and the angles of the principal radii are changed and denoted as $R_{2 X}, R_{2 Y}, S_{2 X}, S_{2 Y}, \theta_{2 X}, \theta_{2 Y}$. By assuming that the displacement is extremely small, the changes in the angles of the principal radii can be neglected, i.e. $\theta_{2 X}=\theta_{1 X}$ and $\theta_{2 Y}=\theta_{1 Y}$, and the lengths of the principal sections of curvature, $S_{2 X}$ and $S_{1 X}, S_{2 Y}$, and $S_{1 Y}$ are related by the law of sines. Then, the surface areas, $\mathrm{d} A_{1}$ and $\mathrm{d} A_{2}$, can be approximated by the product of the lengths of the principal sections of curvature, $S_{1 X}$ and $S_{1 Y}, S_{2 X}$, and $S_{2 Y}$, respectively. By subtracting $\mathrm{d} A_{1}$ from $\mathrm{d} A_{2}$, the net increase in area caused by a small displacement can be derived. By neglecting the second-order term, the net increase in surface area is expressed as

$$
\mathrm{d} \Delta A=\mathrm{d} A_{1} \mathrm{~d} h\left(\frac{1}{R_{1 X}}+\frac{1}{R_{1 Y}}\right)
$$

The net increase in area can also be derived by vector analysis. The finite surface areas, $\mathrm{d} A_{1}$ and $\mathrm{d} A_{2}$, are expressed by the cross product of the surface position vectors. By neglecting the higher order terms and subtracting $\mathrm{d} A_{1}$ from $\mathrm{d} A_{2}$, the net increase in area is derived as follows 


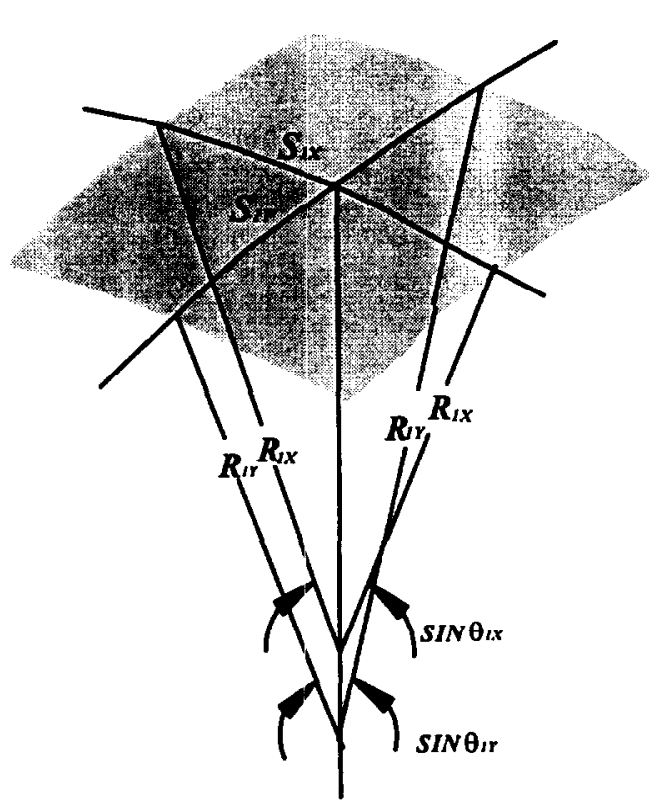

Fig. 1. The relation between curve surface and curvature.

$$
\mathrm{d} \Delta A \approx-\left(\frac{\partial^{2} Z_{h}}{\partial X^{2}}+\frac{\partial^{2} Z_{h}}{\partial Y^{2}}\right) \mathrm{d} A_{1} \mathrm{~d} h
$$

where $Z_{h}$ denotes the position of the surface in the $\mathrm{z}$-direction. The negative sign in eqn (4) indicates the surface curvature is convex. From eqn (2), eqn (3) and eqn (4), the relationship between the capillary pressure and surface curvature is expressed as

$$
\left(P_{1}-P_{2}\right)=-\sigma\left(\frac{\partial^{2} Z_{h}}{\partial X^{2}}+\frac{\partial^{2} Z_{h}}{\partial Y^{2}}\right)
$$

\subsection{The interface velocity}

Although a pressure difference exists across the interface, the velocity of particles located on the interface should be equal. The analysis of Glass ${ }^{11}$ uses a relationship between interface velocity and curvature established by Parlange et $a l^{26}$, with the addition of a perturbation velocity that exists in the interface, to approach the velocity of the interface as follows

$$
U=V-\Gamma\left(\frac{1}{R_{1 X}}+\frac{1}{R_{1 Y}}\right)+\frac{\partial h}{\partial t}
$$

where $U\left(L T^{-1}\right)$ denotes the velocity of the interface, and $V\left(L T^{-1}\right)$ represents the mean velocity of the fluid. The perturbation velocity, determined by the first derivative of the perturbation displacement $h$ with respect to time, $\Gamma\left(L^{2} T\right)$, is defined for the fluid that would spontaneously displace the other by capillary action. In the remainder of this paper, we take this to be the displacing fluid. It is expressed as ${ }^{11}$

$$
\Gamma=\int \frac{\frac{P_{F}}{\rho_{F} g}}{\frac{P_{O}}{\rho_{O} g}} \frac{K \mathrm{~d}\left(\frac{P}{\rho g}\right)}{\theta_{F}-\theta_{O}}
$$

where $K(L / T)$ denotes the hydraulic conductivity, $\theta$ represents the fluid content, $\rho\left(M L^{-3}\right)$ is the density of fluid, $g\left(L T^{-2}\right)$ denotes the gravitational acceleration constant, and the subscripts $O$ and $F$ represent values in front of and in back of the diffusive zone, respectively. $\Gamma$ is important due to the existence of a capillary fringe. Fetter ${ }^{8}$ pointed out that the capillary fringe is very thin, since both the immiscible fluids are liquid, thus $\Gamma$ is neglected in this study, since the density and pressure differences across the diffusive zone are not too large. Therefore, the velocity of the interface is simplified to

$$
U=V+\frac{\partial h}{\partial t}
$$

Darcy's law is generally adopted to describe the flow through non-deformable, porous media within the regime of small Reynolds numbers, where inertial forces are negligible relative to viscous forces. It can be expressed as follows

$$
U_{i}=\frac{-k}{\mu_{i} \theta_{i}}\left(\frac{\partial P_{i}}{\partial Z}+\rho_{i} g\right) i=1,2
$$

where $k\left(L^{2}\right)$ denotes the intrinsic permeability, $\mu\left(M L^{-1} T^{-2}\right)$ represents the viscosity, and the subscripts 1 and 2 are the type of fluid. By assuming that pore volume conducting either fluid is the same, $\theta_{1}=\theta_{2}=\theta_{f}$ is adopted.

If the direction of gravity is assumed to be parallel to the base flow velocity, in view of eqn (7) and eqn (8), the relationships among the pressure, velocity and position at the interface can be expressed as follows

$$
\frac{-k}{\mu_{i} \theta_{f}}\left(\frac{\partial P_{i}}{\partial Z}+\rho_{i} g\right)=V+\frac{\partial h}{\partial t} i=1,2
$$

\section{STOCHASTIC MODEL}

\subsection{Immiscible flow equations}

For two incompressible immiscible fluids (denoted by 1 and 2) having different viscosities and densities within the porous media, the equation of continuity gives

$$
\begin{aligned}
& \nabla \cdot \frac{k}{\mu_{1} \theta_{f}}\left[\nabla\left(P_{1}+\rho_{1} g Z\right)\right]=0 \\
& \nabla \cdot \frac{k}{\mu_{2} \theta_{f}}\left[\nabla\left(P_{2}+\rho_{2} g Z\right)\right]=0
\end{aligned}
$$

eqn (10) is a Laplace-type equation. To find a solution for the flow velocity, the specific boundary conditions must be given. Twelve boundary conditions are required to solve eqn (10). Two boundary conditions have been imposed at the interface between the two fluids. The other conditions are set as the $P_{1}+\rho_{1} g Z$ and $P_{2}+\rho_{2} g Z$, bounded as $X, Y \rightarrow-\infty$ and $\infty$, respectively, to simplify the problem. One of the interface conditions is cxpressed as eqn (5). The other interface condition reflects the continuity of velocity 
at the interface

$$
\left.\frac{k}{\mu_{1} \theta_{f}}\left(\frac{\partial P_{1}}{\partial Z}+\rho_{1} g\right)\right|_{Z=Z_{h}}=\left.\frac{k}{\mu_{2} \theta_{f}}\left(\frac{\partial P_{2}}{\partial Z}+\rho_{2} g\right)\right|_{Z=Z_{h}}
$$

If the natural logarithm of intrinsic permeability, $\ln k$, the pressure, $P$, and the position of the interface, $H$, are assumed to be second-order stationary stochastic processes, eqn (10) and eqn (11) become stochastic partial differential equations. $\ln k, P$ and $H$ can be decomposed into mean values and fluctuations of these values around their means, i.e.

$$
\begin{aligned}
& \ln k=B+b, B=E[\ln k], E[b]=0 \\
& P_{i}=\bar{P}_{i}+P_{i}{ }^{\prime}, \bar{P}_{i}=E\left[P_{i}\right], E\left[P^{\prime}\right]=0 \\
& H=\bar{H}+h, \bar{H}=E[H], E[h]=0
\end{aligned}
$$

where $B, \bar{P}_{i}$ and $\bar{H}$ denote the means of $\ln k, P_{i}$ and $H$, and $b, P_{i}^{\prime}$ and $h$ represent the perturbations of $\ln k, P_{i}$ and $H$, respectively. Substituting eqn (12) into the governing eqn (10), yields

$$
\begin{aligned}
& \frac{\partial^{2}\left(\bar{P}_{i}+P_{i}{ }^{\prime}\right)}{\partial X^{2}}+\frac{\partial^{2}\left(\bar{P}_{i}+P_{i}{ }^{\prime}\right)}{\partial Y^{2}}+\frac{\partial^{2}\left(\bar{P}_{i}+P_{i}{ }^{\prime}\right)}{\partial Z^{2}} \\
& +\frac{\partial(B+b)}{\partial X} \frac{\partial\left(\bar{P}_{i}+P^{\prime}\right)}{\partial X}+\frac{\partial(B+b)}{\partial Y} \frac{\partial\left(\bar{P}_{i}+P^{\prime}\right)}{\partial Y} \\
& +\frac{\partial(B+b)}{\partial Z} \frac{\partial\left(\bar{P}_{i}+P^{\prime}\right)}{\partial Z}+\rho_{i} g \frac{\partial(B+b)}{\partial Z}=0
\end{aligned}
$$

By subtracting the mean equation from eqn (13), a perturbation equation is obtained as

$$
\begin{aligned}
& \frac{\partial^{2} P_{1}^{\prime}}{\partial X^{2}}+\frac{\partial^{2} P_{1}^{\prime}}{\partial Y^{2}}+\frac{\partial^{2} P_{1}^{\prime}}{\partial Z^{2}}+\frac{\partial b}{\partial Z} \frac{\partial \bar{P}_{1}}{\partial Z}+\rho_{1} g \frac{\partial b}{\partial Z}=0 \\
& \frac{\partial^{2} P_{2}^{\prime}}{\partial X^{2}}+\frac{\partial^{2} P_{2}^{\prime}}{\partial Y^{2}}+\frac{\partial^{2} P_{2}^{\prime}}{\partial Z^{2}}+\frac{\partial b}{\partial Z} \frac{\partial \bar{P}_{2}}{\partial Z}+\rho_{2} g \frac{\partial b}{\partial Z}=0
\end{aligned}
$$

For the boundaries in the interface, the perturbation equations can be derived by substituting eqn (12) into eqn (11) and eqn (5), then subtracting the mean equation from the perturbation equation. Therefore,

$$
\begin{gathered}
\frac{e^{B}}{\mu_{1} \theta_{f}}\left[\frac{\partial P_{1}^{\prime}(\bar{H})}{\partial Z}+b \frac{\partial \bar{P}_{1}(\bar{H})}{\partial Z}+\rho_{1} g b\right] \\
=\frac{e^{B}}{\mu_{2} \theta_{f}}\left[\frac{\partial P_{2}^{\prime}(\bar{H})}{\partial Z}+b \frac{\partial \bar{P}_{2}(\bar{H})}{\partial Z}+\rho_{2} g b\right] \\
P_{1}^{\prime}(\bar{H})-P_{2}^{\prime}(\bar{H})=-\sigma\left(\frac{\partial^{2} h}{\partial X^{2}}+\frac{\partial^{2} h}{\partial Y^{2}}\right) \\
+\left(J_{2}-J_{1}\right)-\left(\rho_{2}-\rho_{1}\right) g h
\end{gathered}
$$

where $J_{i}=-\frac{V \mu_{I} \theta_{f}}{e^{B}}$

After perturbătion analysis, three dependent variables,
$P_{1}{ }^{\prime}, P_{2}{ }^{\prime}$ and $h$ exist in eqn (14), as the mean pressure gradient is assumed to be constant. Therefore, eqn (9) is included in the boundary conditions. The perturbation equation of eqn (9) is thus derived as follows

$$
\frac{e^{B}}{\mu_{i} \theta_{f}}\left(\frac{\partial P_{i}^{\prime}}{\partial Z}+J_{i} b\right)=-\frac{\mathrm{d} h}{\mathrm{~d} t}
$$

\subsection{Spectrum analysis}

The assumption of statistical stationarity is frequently employed in stochastic hydrogeology. The stationarity assumption enables a varicty of analytical techniques, c.g. Fourier transformation theory, to be used to solve stochastic flow and transport problems. Herein, the intrinsic permeability and the position of the interface in space are assumed to be stationarities. Thus, derived perturbation variables can be represented by the Fourier-Stieltjes Integral ${ }^{34}$ :

$$
\begin{aligned}
& b=\int_{-\infty}^{\infty} \int_{-\infty}^{\infty} \int_{-\infty}^{\infty} e^{i\left(w_{1} X+w_{2} Y+w_{3} Z\right)} \mathrm{d} Z_{b}(\mathbf{w}) \\
& h=\int_{-\infty}^{\infty} \int_{-\infty}^{\infty} e^{i\left(w_{1} X+w_{2} Y\right)} \mathrm{d} Z_{H}(\mathbf{w})
\end{aligned}
$$

where $w_{i}$ denotes a component $i$ of the wave number vector $\mathbf{w}$, and $\mathrm{d} Z_{h}$ and $\mathrm{d} Z_{b}$ represent the complex Fourier amplitudes of the fluctuations $b$ and $h$ over wave number space w. Although the intrinsic permeability is stationarity, the perturbation term of pressure always varies according to the imposed boundary conditions $^{20} . \mathrm{Li}$ and Mclaughlin ${ }^{20}$ represented the nonstationarity perturbation term as a stochastic integral, expanded in terms of transfer functions $\phi_{i}(X, w)$ over the stationarity increment $\mathrm{d} Z_{b}(w)$

$$
P_{i}^{\prime}=\int_{-\infty}^{\infty} \int_{-\infty}^{\infty} \int_{-\infty}^{\infty} \phi_{i}(\mathbf{X}, \mathbf{w}) \mathrm{d} Z_{b}(\mathbf{w})
$$

By assuming that the perturbation term of pressure is nonstationary only in the Z-direction and does not vary in other directions, the transfer functions can be simplified as follows

$$
\phi_{i}(\mathbf{X}, \mathbf{w})=\bar{\phi}_{i}\left(Z, w_{3}\right) e^{i\left(w_{1} X+w_{2} Y\right)}
$$

Substituting eqn (18), eqn (19), eqn (20) and eqn (21) into eqn (14), eqn (15), eqn (16) and eqn (17), and neglecting the quadratic terms of perturbation yield

$$
\begin{aligned}
& \bar{\phi}_{1}=C_{2} e^{r \bar{H}}+i \frac{w_{1} J_{1}}{w_{3}^{2}+r^{2}} e^{i w_{3} \bar{H}} \\
& \bar{\phi}_{2}=C_{3} e^{r \bar{H}}+i \frac{w_{1} J_{2}}{w_{3}^{2}+r^{2}} e^{i w_{3} \bar{H}} \\
& \mathrm{~d} Z_{h}=\frac{\int \bar{\phi}_{1}(\bar{H})-\bar{\phi}_{2}(\bar{H}) \mathrm{d} Z_{b}}{\left[-\sigma r^{2}+\left(J_{2}-J_{1}\right)-\left(\rho_{2}-\rho_{1}\right) g\right]} \\
& \frac{\partial \bar{\phi}_{2}(\bar{H})}{\partial Z}-\frac{\partial \bar{\phi}_{1}(\bar{H})}{\partial Z}+\left(J_{2}-J_{1}\right) e^{i\left(w_{3} \bar{H}\right)} \\
& =M\left(\frac{\partial \bar{\phi}_{2}(\bar{H})}{\partial t}-\frac{\partial \bar{\phi}_{1}(\bar{H})}{\partial t}\right)
\end{aligned}
$$


where $C_{2}$ and $C_{3}$ are undetermined coefficients, and

$$
\begin{aligned}
& M=\frac{\left(\mu_{2}-\mu_{1}\right) \theta_{F}}{\left[-\sigma r^{2}+\left(J_{2}-J_{1}\right)-\left(\rho_{2}-\rho_{1}\right) g\right] e^{b}} \\
& r^{2}=w_{1}^{2}+w_{2}^{2}
\end{aligned}
$$

Cary et al. $^{3}$ provided the positions of the front between two immiscible fluids versus time. They observed fluids infiltrating into both the tops and bottoms of columns in a laboratory experiment and recorded the time required for all the fluid fronts to move. A linear relationship between the fluid fronts and time is established after several hours. Therefore, the mean position of the interface $\bar{H}$ is simplified as

$$
\bar{H}=V t
$$

By substituting eqn (25) into eqn (22) and eqn (24), $C_{2}$ and $C_{3}$ can be determined as where $\xi$ denotes the length of the separation vector, and $\mathrm{d} Z_{h}^{*}$ represents the complex conjugate of $\mathrm{d} Z_{h}$. The expectation of $E\left[\mathrm{~d} Z_{h} \mathrm{~d} Z_{h}^{*}\right]$ can be derived by substituting eqn (26) and eqn (27) into eqn (22) and eqn (23). The above results include the spectrum of the logarithm of intrinsic permeability. The spectrum of the logarithm of intrinsic permeability derived by $\mathrm{Yeh}^{35}$ for threedimensional environments is adopted, and expressed as follows

$$
s_{b b}=\frac{\sigma_{b}^{2} \lambda^{3}}{\pi^{2}\left[1+\lambda^{2}\left(w_{1}^{2}+w_{2}^{2}+w_{31}^{2}\right)\right]^{2}}
$$

where $\sigma_{b}$ denotes the variance of the logarithm of intrinsic permeability, and $\lambda$ represents the correlation length. Substituting eqn (29) into eqn (28) and expressing the autocovariance function in spherical coordinates, eqn (28) can

$$
\begin{aligned}
& C_{2}=\left[-\frac{r^{2} V^{2} \mu_{1}\left[\frac{e^{B} r^{2}}{C_{A}}+\frac{e^{B} B_{O}}{C_{A}}\right]+V^{2} \mu_{1} \theta_{f}\left(B_{M}-1\right)\left(r^{2}-w_{3}^{2}\right)}{\left(w_{3}^{2}+r^{2}\right) e^{B}\left(-\frac{V r\left[e^{B} r^{2}+e^{B} B_{0}\right]}{C_{A} \theta_{f}}-r V\left(B_{M}-1\right)+i w_{3} V\right)}\left(e^{\left(i w_{3} V+r V\right)_{t}}-e^{\frac{2 B_{M} V r t}{B_{M}+1}+\frac{\left[e^{B} r^{2}+e^{B} B_{O}\right] V r t}{\left(1+B_{M}\right) C_{A} \theta_{f}}}\right)\right] \\
& -\frac{i w_{3} J_{1}}{w_{3}^{2}+r^{2}} e^{\frac{2 B_{M} V r t}{B_{M}+1}+\frac{\left[e^{B} r^{2}+e^{B} B_{O}\right] V r t}{C_{A}\left(B_{M}+1\right) \theta_{f}}} \\
& C_{3}=\left[-\frac{r^{2} V^{2} \mu_{2}\left[\frac{e^{B} r^{2}}{C_{A}}+\frac{e^{B} B_{O}}{C_{A}}\right]+V^{2} \mu_{2} \theta_{f}\left(B_{M}-1\right)\left(r^{2}-w_{3}^{2}\right)}{\left(w_{3}^{2}+r^{2}\right) e^{B}\left(-\frac{V r\left[e^{B} r^{2}+e^{B} B_{0}\right]}{C_{A} \theta_{f}}-r V\left(B_{M}-1\right)+i w_{3} V\right)}\left(e^{\left(i w_{3} V+r V\right) t}-e^{\frac{2 B_{M} V r t}{B_{M}+1}+\frac{\left[e^{B} r^{2}+e^{B} B_{O}\right] V r t}{\left(1+B_{M}\right) C_{A} \theta_{f}}}\right)\right] \\
& -\frac{i w_{3} J_{2}}{w_{3}^{2}+r^{2}} e^{\frac{2 B_{M} V r t}{B_{M}+1}+\frac{\left[e^{B} r^{2}+e^{B_{o}}\right] V r t}{C_{A}\left(B_{M}+1\right) \theta_{f}}}
\end{aligned}
$$

where

$$
\begin{aligned}
B_{M} & =\begin{array}{l}
\mu_{2} \\
\mu_{1}
\end{array} \\
C_{A} & =\frac{V \mu_{1}}{\sigma} \\
B_{O} & =\frac{e^{B}\left(\rho_{2}-\rho_{1}\right) g}{\sigma}
\end{aligned}
$$

eqn (23) is the spectrum relationship between the position of the interface and the intrinsic permeability in the frequency domain. Based on time-series analysis ${ }^{21,34}$, the auto-covariance function for the position of the interface can be represented as

$$
R(\xi)=\int e^{\bar{\nu} \vec{w} \cdot \vec{\xi}} E\left[\mathrm{~d} Z_{h} \mathrm{~d} Z_{h}^{*}\right]
$$

be rewritten as

$$
\begin{aligned}
& R(\xi)=\frac{\lambda^{3} \sigma_{b}^{2}}{\pi^{2}} \int_{0}^{\infty} \int_{0}^{2 \pi} \int_{0}^{\pi} \\
& \frac{e^{i \omega \cos \theta} B^{*} \sin \theta}{\left[\frac{w^{2}\left(1-\sin ^{2} \theta \cos ^{2} \partial\right)}{B_{M} C_{A}}+\frac{\theta_{f}}{e^{B}}\left(1-\frac{1}{B_{M}}\right)+\frac{B_{0}}{e^{B} C_{A} B_{M}}\right]^{2}\left(1+\lambda^{2} \omega^{2}\right)^{2}} \mathrm{~d} \theta \mathrm{d} \alpha \mathrm{d} w
\end{aligned}
$$

The coordinate transformation relationships developed by Bakr $e t$ al. ${ }^{1}$ are given in Fig. 2. The expression of $B^{*}$ is given in Appendix A. The variance of the position of the interface can be calculated from eqn (30) by setting $\xi$ equal to zero. Based on stochastic theory, variance is defined as the difference between a random value of a variable and its mean value. The variance increases as the instability of the 


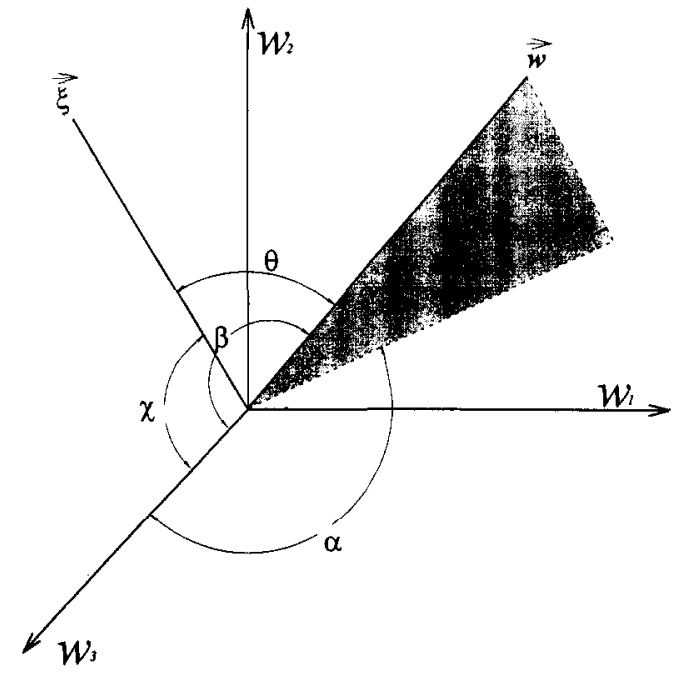

Fig. 2. Coordinate system of wave number and separation vectors relations.

wetting front increases. Thus, the variance of the interface position can be viewed as a criterion to define an unstable wetting front. Eqn (30) is not easy to integrate analytically. Herein, the oscillatory technique developed by Davies et al..$^{7}$ is adopted to solve this problem.

\section{RESULTS AND DISCUSSION}

The exponential function, $e^{\bar{G} t}$, in eqn (30) (see Appendix A) is a function of time. Thus, $\bar{G}$ can be viewed as a criterion to predict whether or not an unstable interface occurs in porous media. The unstable interface occurs as $\bar{G}$ becomes greater than zero, because the auto-covariance calculated from eqn (30) increases over time $t$. For the stable interface, autocovariance decreases over time as $\bar{G}$ becomes less than zero. The terms that determine whether $\bar{G}$ is positive or negative can be rearranged as follows

$$
V \theta_{f}\left(\mu_{2}-\mu_{1}\right)-r^{2} e^{B} \sigma+\left(\rho_{2}-\rho_{1}\right) g e^{B}
$$

where the first term and third term are constant values, and the second term is varied with $r$ and always greater than zero. The fluid properties must satisfy the following equation to allow $\bar{G}$ to have a negative value

$$
V \theta_{f}\left(\mu_{2}-\mu_{1}\right)+\left(\rho_{2}-\rho_{1}\right) g e^{B}<0
$$

eqn (32) is the criterion of a stable interface. The stability depends on the fluid flow velocity and fluid properties, and the critical velocity, $V_{c}$, can be obtained by setting the equation equal to zero. The relationship between $\mu_{2}<\mu_{1}$ and $\rho_{2}<\rho_{1}$ define the conditions of absolute stability as the fluid flow direction opposes the gravitational direction (upward displacement, i.e. fluid 1 displaces fluid 2), while $\mu_{2}>\mu_{1}$ and $\rho_{2}<\rho_{1}$ are the conditions for stable flow as the fluid direction and gravitational direction are the same (i.e. fluid 2 displaces fluid 1 , and the velocity has a negative value). Table 1 lists the other conditions of stability for the combination of different fluid propertics. Those results correspond to those of Kueper ${ }^{18}$.

Three dimensionless parameters, $B_{M}, C_{A}$ and $B_{O}$, appear in eqn (30). $B_{M}$ is known as the mobility ratio, defined as the mobility of the displacing fluid, divided by the mobility of the displaced fluid. If the mobility ratio is greater than one, it favours the flow of the displacing fluid, whereas if this ratio is less than one, it favours the flow and recovery of displaced fluid. $C_{A}$ is the capillary number, defined as the ratio of viscous and capillary forces. Larson et al. ${ }^{19}$ compiled numerous experimental data of oil mobilization by water flooding, indicating that residual saturation correlates strongly with the value of the capillary number. Hunt et $a l^{16}$ also indicated that residual oil can be displaced if the capillary number exceeds $2 \times 10^{-5} . B_{0}$ is the Bond number, i.e. the ratio of gravitational and surface tension forces.

To understand the relations between the interface growth and the dimensionless parameters, the non-dimensional analysis of $R(0) / \sigma_{b}^{2}$ is carried out. The cases of unconditional instability in Table 1 are selected. The relations $B_{M}>1, B_{0}>1$ and $B_{M}<1, B_{0}>1$ are adopted for upward and downward displacements, respectively. Other physical properties used in the analysis are shown in Table 2. In the upward displacement case, the profiles of $R(0) / \sigma_{b}^{2}$ versus $B_{M}$ for capillary numbers equal to 5 and 10 are shown in Fig. 3a and b, respectively. According to these results, $R(0) / \sigma_{b}^{2}$ decreases as the mobility ratio decreases. Notably, that increasing the Bond number results in a shallow slope, while a lower Bond number yields a steeper slope. Fig. $4 \mathrm{a}$ and $\mathrm{b}$ display the profiles of $R(0) / \sigma_{b}^{2}$ versus $C_{A}$ for mobility ratios equal to 5 and 10 , respectively. The $R(0) / \sigma_{b}^{2}$ value initially increases with increasing $C_{A}$ while the slope of the profile gradually decreases. The $C_{A}$ is proportional to $V, \mu_{1}$ and $\sigma^{-1}$. Thus, the perturbation in the interface increases with increases of the mean flow velocity as the physical properties are fixed. Therefore, this result

Table 1. The fluid properties for stable displacement

\begin{tabular}{lccc}
\hline \multicolumn{2}{c}{ Upward displacement } & \multicolumn{2}{c}{ Downward displacement } \\
\hline Fluid properties & Stable condition & Fluid properties & Stable condition \\
\hline$\rho_{1}>\rho_{2}, \mu_{1}<\mu_{2}$ & $V<V_{C}$ & $\rho_{2}>\rho_{1}, \mu_{2}<\mu_{1}$ & Always unstable \\
$\rho_{1}>\rho_{2}, \mu_{1}>\mu_{2}$ & Always stable & $\rho_{2}>\rho_{1}, \mu_{2}<\mu_{1}$ & $V>V_{C}$ \\
$\rho_{1}<\rho_{2}, \mu_{1}<\mu_{2}$ & Always unstable & $\rho_{2}<\rho_{1}, \mu_{2}<\mu_{1}$ & $V<V_{C}$ \\
$\rho_{1}<\rho_{2}, \mu_{1}>\mu_{2}$ & $V>V_{C}$ & $\rho_{2}<\rho_{1}, \mu_{2}<\mu_{1}$ & Always stable \\
\hline
\end{tabular}


Table 2. Physical condition of porous media and fluids

\begin{tabular}{cc}
\hline Relation length & $100 \mathrm{~m}$ \\
Mean value of intrinsic permeability & $100^{-7} \mathrm{~m}^{2}$ \\
Mean value of velocity & $6.7 \times 10^{-3} \mathrm{~m} /$ day \\
Porosity & 0.4 \\
Time & 1.0 day \\
\hline
\end{tabular}

clearly suggests that the perturbation of the interface is not affected by the capillary number when the mean velocity is sufficiently larger than the critical velocity. The profiles of $R(0) / \sigma_{b}^{2}$ versus $B_{0}$ for mobility ratios equal to 5 and 10 are shown in Fig. 5a and b, respectively. The above results demonstrate that $R(0) / \sigma_{b}^{2}$ decreases with an increasing $B \_\{0\}$. The Bond number is proportional to the mean intrinsic permeability and $\sigma^{-1}$. Thus, the unstable interface front is more easily formed in the sandy formation than in the clayey formation. On the other hand, the perturbation of the interface increases with an increasing tension of interface.

If the fluid flow is driven by gravitational force, the interface of the two fluids moves in the gravitational direction. In downward displacement, the relations between $R(0) / \sigma_{b}^{2}$ and the dimensionless parameter are also investigated. The flow
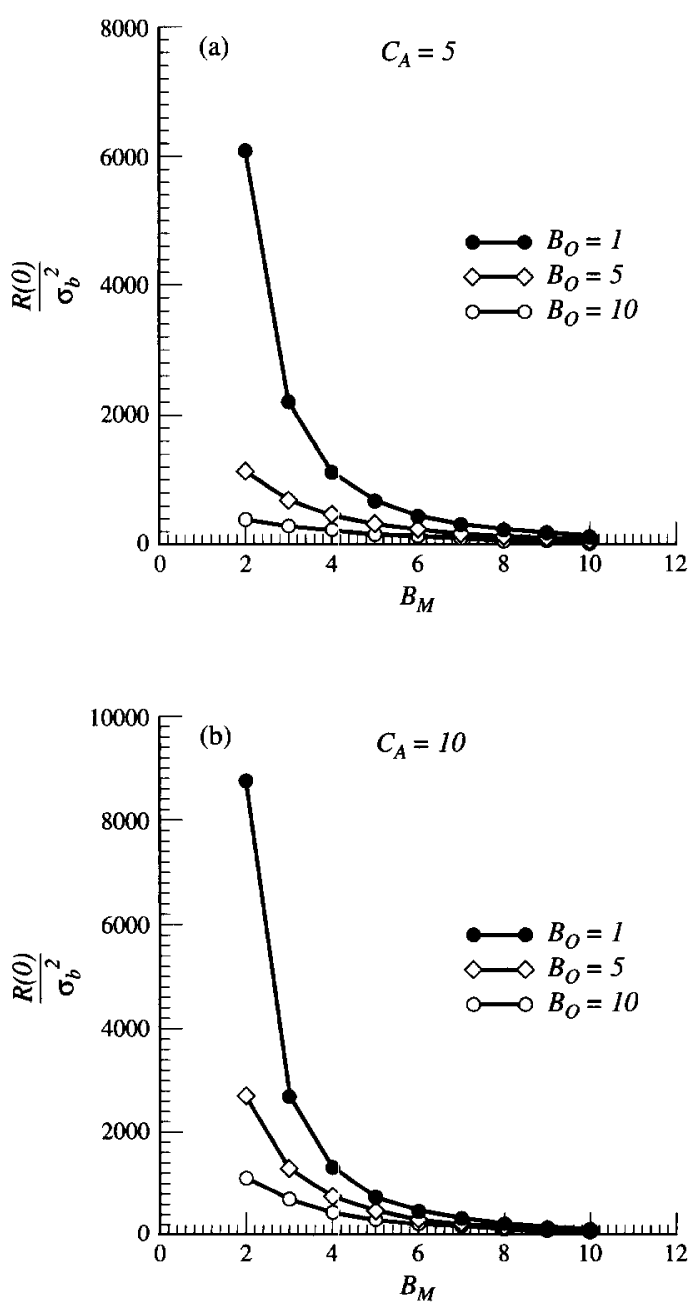

Fig. 3. The variance of unstable interface versus mobility ratio in the upward fluid flow system. The relation with different capillary numbers $C_{A}=1$ and $C_{A}=10$ are shown in (a), (b) respectively.
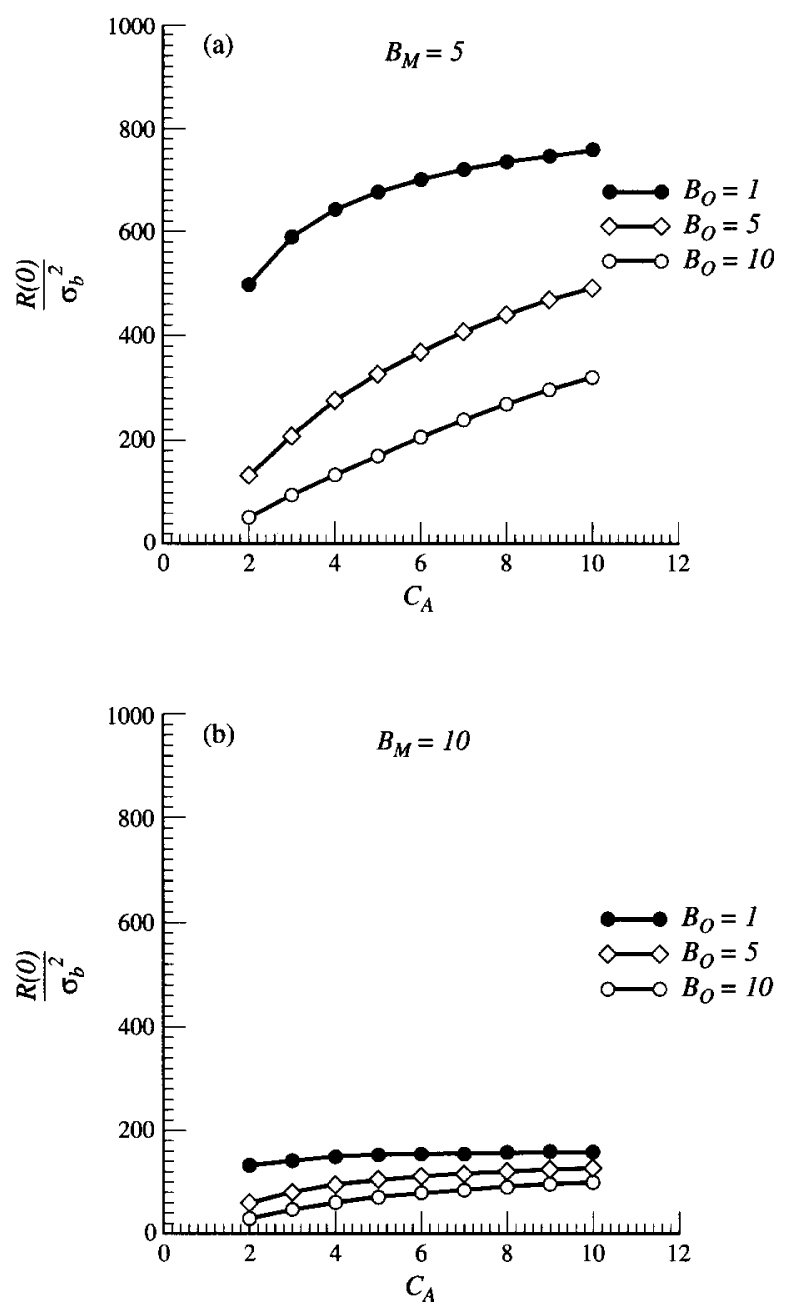

Fig. 4. The variance of unstable interface versus capillary number in the upward fluid flow system. The relation with different mobility ratios $B_{M}=5$ and $B_{M}=10$ are shown in (a), (b) respectively.

velocity, $V$, and capillary number, $C_{A}$, are defined as negative values, since the fluid flow direction is opposite to the reference coordinate. The profiles of $R(0) / \sigma_{b}^{2}$ versus $B_{M}, C_{A}$, $B_{O}$ are shown in Fig. 6, Fig. 7, and Fig. 8, respectively. The $R(0) / \sigma_{b}^{2}$ increases with increasing $B_{M}$ and decreases with increasing $C_{A}$ and $B_{O}$ respectively. In this case, the mobility ratio is equal to the inverse of $B_{M}$, since fluid 2 displaces fluid 1 . Thus the $R(0) / \sigma_{b}^{2}$ decreases as the mobility ratio increases. The negative sign on the capillary number results because the flow direction is opposite to the reference coordinate; the capillary number of a fluid with a large mean velocity is less than that of a fluid with a small mean velocity. Therefore, the $R(0) / \sigma_{b}^{2}$ increases with increases in mean velocity as the fluid properties are fixed. This result is consistent with the previous discussion of the upward displacement case.

For a horizontal flow case, the Bond number is set to be zero and, hence, the gravitational force is neglected in eqn (30). Fig. 9 displays the profiles of $R(0) / \sigma_{b}^{2}$ versus capillary numbers. The result shows that the generation of an unstable interface is not affected by capillary number. Therefore, eqn (30) is simplified by setting the capillary number equal to 

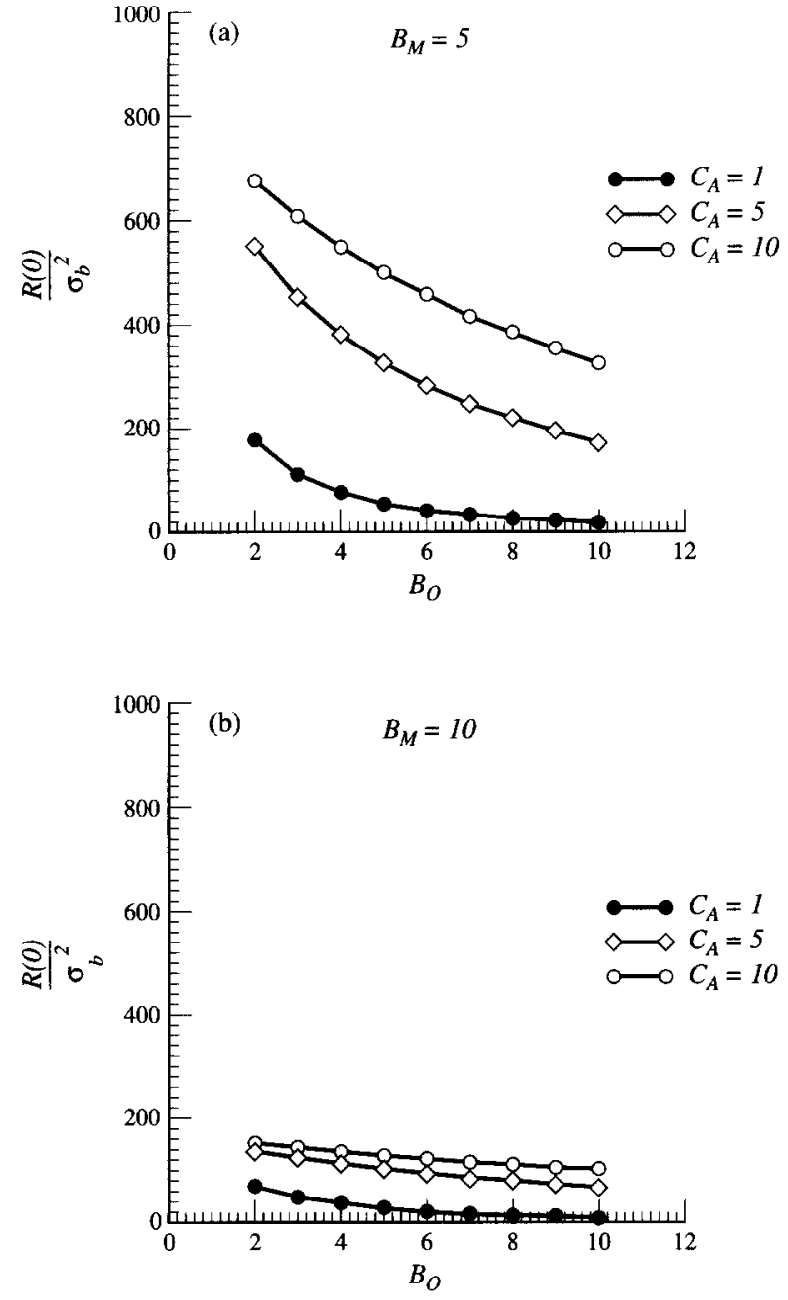

Fig. 5. The variance of unstable interface versus bond number in the upward fluid flow system. The relation with different mobility ratios $B_{M}=5$ and $B_{M}=10$ are shown in (a), (b) respectively.

infinity. The results are shown in Fig. 10. The change of $R(0) / \sigma_{b}^{2}$ decreases with an increasing mobility ratio, when the mean velocity is small. Nevertheless, a critical mobility ratio is apparent in Fig. 10, when the mean velocity is large. The $\rho_{1}>\rho_{2}, \mu_{1}>\mu_{2}$ value decreases with an increasing mobility ratio, if the mobility ratio is less than the critical mobility ratio. When the mobility ratio becomes larger than the critical mobility ratio, $R(0) / \sigma_{b}^{2}$ increases as the mobility ratio increases. In other words, the generation of an unstable interface not only depends on the mobility ratio, but is also affected by the mean flow velocity.

\section{CONCLUSIONS}

This study presents a two-phase stochastic flow model of the variance of interface displacement. Parameters that control the interface instability of the wetting front are investigated from the auto-covariance function of the interface displacement. Computational results indicate that the covariance of interface displacement is a function of mobility ratio, capillary number and Bond number. The directions of flow and
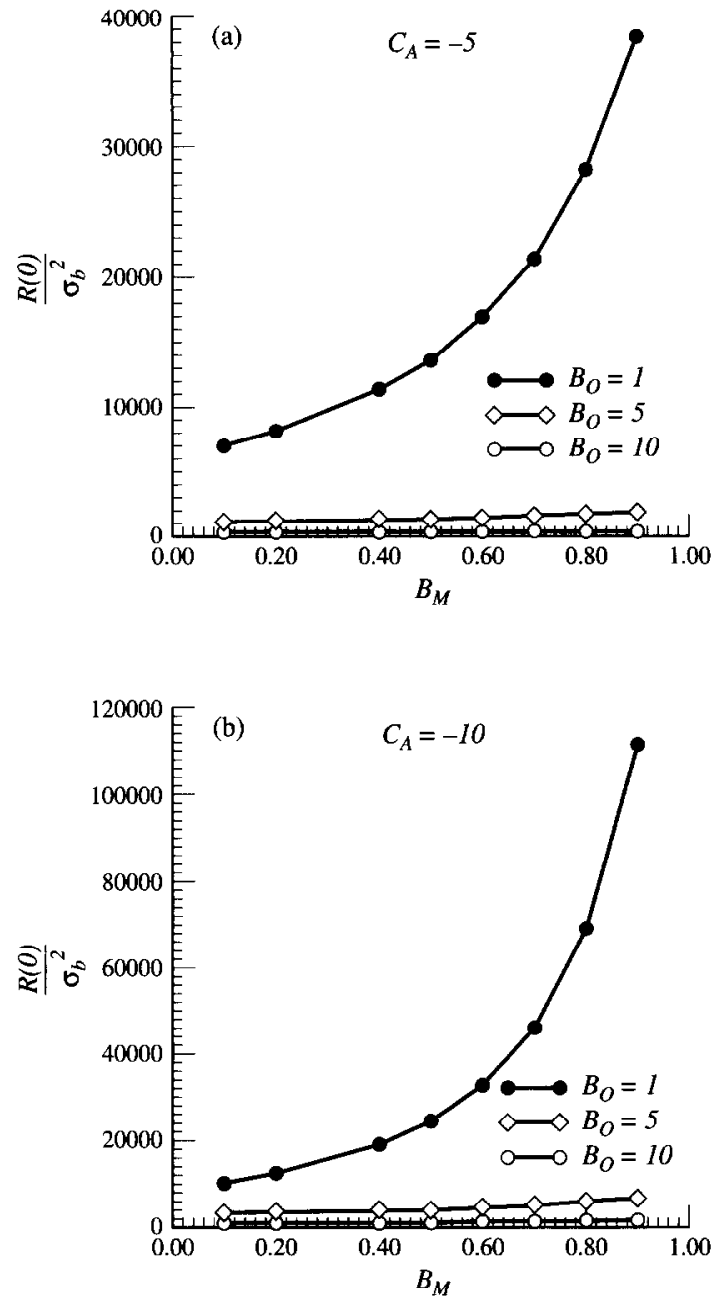

Fig. 6. The variance of unstable interface versus mobility ratio in the downward fluid flow system. The relation with different capillary numbers $C_{A}=-5$ and $C_{A}=-10$ are shown in (a), (b) respectively.

velocity of the fluids are also important factors that control the instability of the wetting front. Based on results obtained from front instability, we can conclude the following:

1. An unstable interface between two immiscible fluids is governed by the fluid properties, such as viscosity and density. The conditions of stability differ with the direction of fluid movement and the combination of viscosity and density. The relations of $\rho_{1}>\rho_{2}, \mu_{1}>\mu_{2}$ and $\rho_{1}<\rho_{2}, \mu_{1}<\mu_{2}$ (fluid 1 displaces fluid 2) are the fluid properties defining the absolute stable and unstable conditions, respectively, for the upward displacement case. The relations of $\rho_{1}<\rho_{2}, \mu_{1}>\mu_{2}$ and $\rho_{1}>\rho_{2}, \mu_{1}<\mu_{2}$ (fluid 2 displaces fluid 1) are the fluid properties defining the absolute stable and unstable conditions, respectively, for the downward displacement case. The other conditions of stability for different combinations of fluid properties depend on whether the velocity exceeds a critical velocity. The critical velocity is a function of fluid properties and mean intrinsic permeability. 

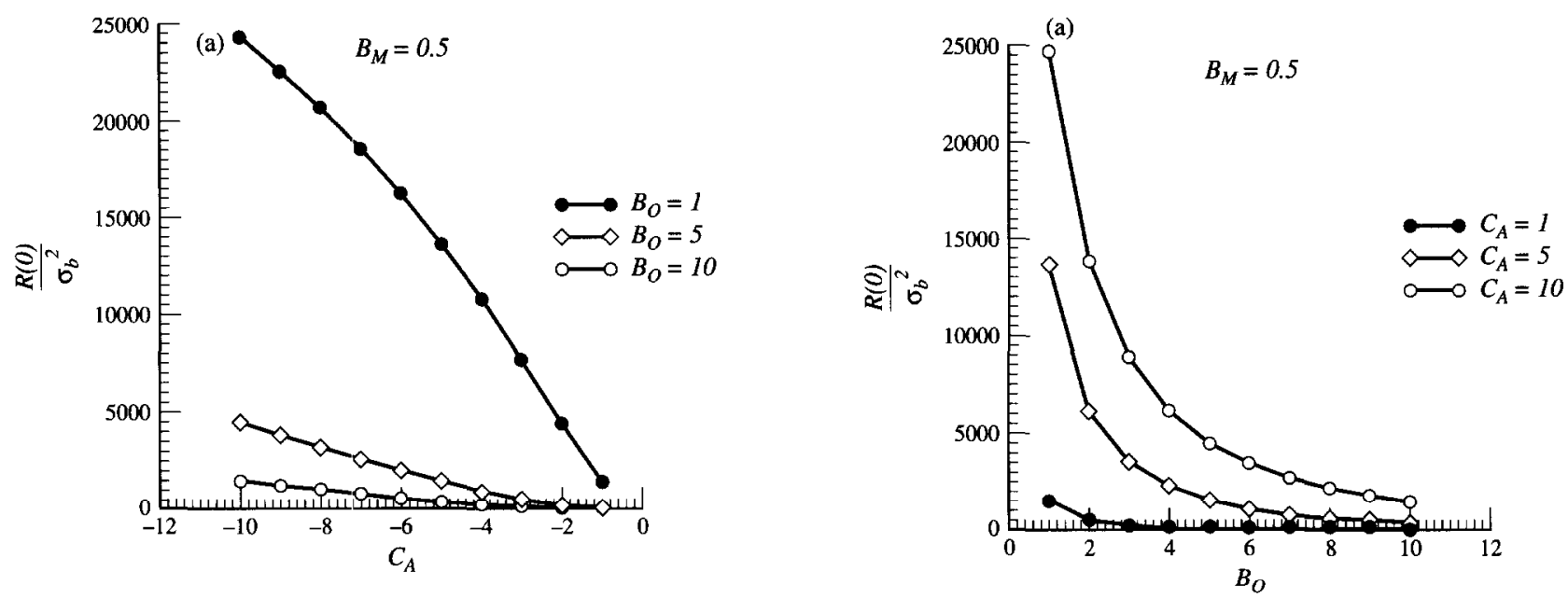

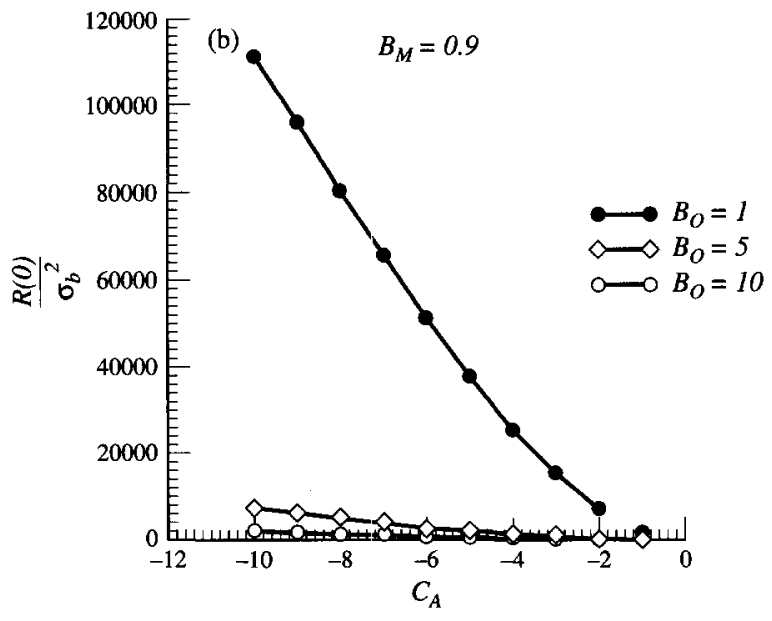

Fig. 7. The variance of unstable interface versus capillary number in the downward fluid flow system. The relation with different mobility ratios $B_{M}=0.5$ and $B_{M}=0.9$ are shown in (a), (b) respectively.

2. The mobility ratio, capillary number and Bond number are three important, dimensionless parameters that affect the interface instability. These results demonstrate that regardless of whether the interface is displaced upward or downward, the variance of an unstable interface decreases with an increasing mobility ratio, increases with an increasing capillary number, and decreases with an increasing Bond number.

3. The capillary number is insignificant in the creation of an unstable interface for the case of horizontal fluid flow. The relation of $\mu_{2}>\mu_{1}$ (fluid 1 displaces fluid 2 ) is always unstable for horizontal displacement. The tendency of the profile of $R(0) / \sigma_{b}^{2}$ versus mobility ratio depends on mean velocity. Also, $R(0) / \sigma_{b}^{2}$ decreases with increasing mobility ratio as fluids flow through porous media with a small mean velocity. A critical mobility ratio exists as fluids flow through porous media with a large velocity. The $R(0) / \sigma_{b}^{2}$ value decreases with an increasing mobility ratio when the mobility ratio is less than the critical

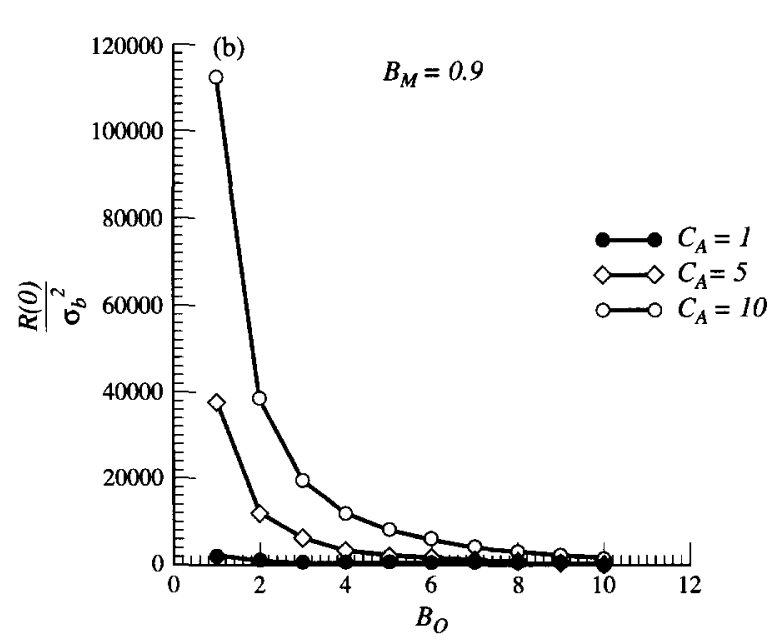

Fig. 8. The variance of unstable interface bond number in the downward fluid flow system. The relation with different mobility ratios $B_{M}=0.5$ and $B_{M}=0.9$ are shown in (a), (b) respectively.

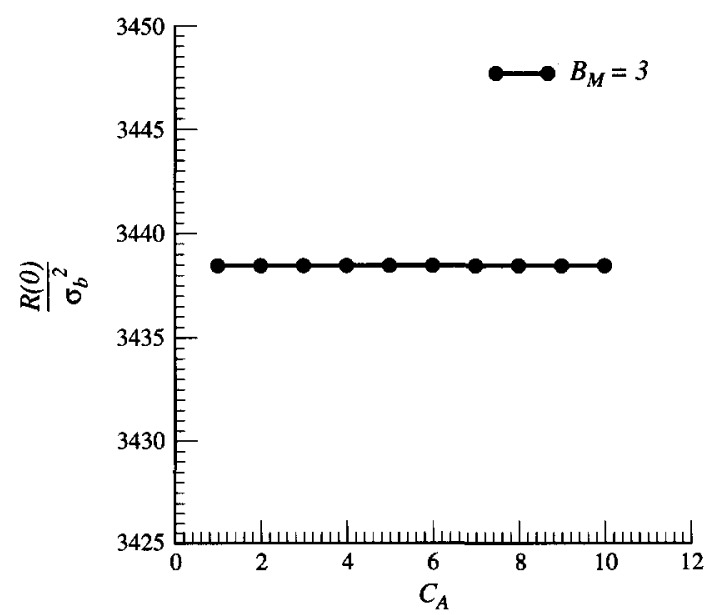

Fig. 9. The variance of unstable interface versus capillary number in the horizontal fluid flow system as $B_{M}=3$. 


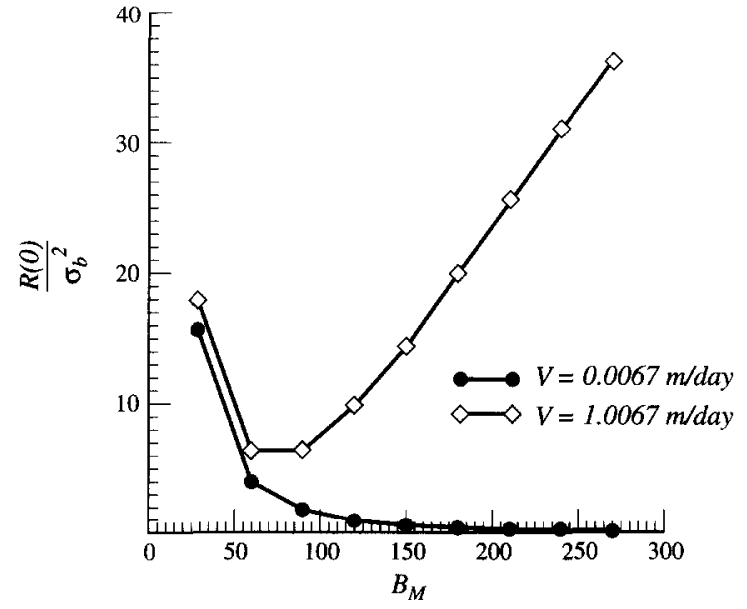

Fig. 10. Variance of unstable interface versus the mobility ratio in the horizontal flow system with different velocities.

mobility ratio. In addition, it increases with increasing mobility ratio when the mobility ratio exceeds the critical mobility ratio.

\section{ACKNOWLEDGEMENTS}

The authors would like to thank the National Science Council of the Republic of China for financially supporting this work under Contract No. NSC-83-0409-b-197-002.

\section{APPENDIX A}

$$
\begin{aligned}
& \left.\quad-2 \bar{H} \bar{F} \sin (w \sin \theta \cos \alpha V t)] e^{\bar{G} t}\right\} \\
& \vec{E}=\left[\frac{w^{2}\left(1-\sin ^{2} \theta \cos ^{2} \alpha\right)^{2}}{C_{A}}+\frac{\left(1-\sin ^{2} \theta \cos ^{2} \alpha\right) B_{O}}{C_{A} e^{B}}\right. \\
& \left.+\frac{\theta_{f}\left(B_{M}-1\right)\left(1-2 \sin ^{2} \theta \cos ^{2} \alpha\right)}{e^{B}}\right] \\
& {\left[\frac{\sin \theta \cos \alpha}{\frac{\left(1-\sin ^{2} \theta \cos ^{2} \alpha\right)}{\left(B_{M}+1\right)^{2}}\left[\left[\frac{w^{2} e^{B}\left(1-\sin ^{2} \theta \cos ^{2} \alpha\right)}{\theta_{f} C_{A}}+\frac{B_{O}}{\theta_{f} C_{A}}+\left(B_{M}-1\right)\right]^{2}+\sin ^{2} \theta \cos ^{2} \alpha\right]}\right]}
\end{aligned}
$$$$
\bar{F}=\left[\frac{W^{2}\left(1-\sin ^{2} \theta \cos ^{2} \alpha\right)^{2}}{C_{A}}+\frac{\left(1-\sin ^{2} \theta \cos ^{2} \alpha\right) B_{O}}{C_{A} e^{B}}+\frac{\theta_{f}\left(B_{M}-1\right)\left(1-2 \sin ^{2} \theta \cos ^{2} \alpha\right)}{e^{B}}\right]
$$$$
\left[\frac{\left[\frac{\sqrt{\left(1-\sin ^{2} \theta \cos ^{2} \alpha\right)}}{\left(B_{M}+1\right)}\left[\frac{w^{2} e^{B}\left(1-\sin ^{2} \theta \cos ^{2} \alpha\right)}{\theta_{f} C_{A}}+\frac{B_{O}}{\theta_{f} C_{A}}+\left(B_{M}-1\right)\right]\right]}{\frac{\left(1-\sin ^{2} \theta \cos ^{2} \alpha\right)}{\left(B_{M}+1\right)^{2}}\left[\left[\frac{w^{2} e^{B}\left(1-\sin ^{2} \theta \cos ^{2} \alpha\right)}{\theta_{f} C_{A}}+\frac{B_{O}}{\theta_{f} C_{A}}+\left(B_{M}-1\right)\right]^{2}+\sin ^{2} \theta \cos ^{2} \alpha\right]}\right]
$$$$
\bar{H}=\frac{\sin \theta \cos \alpha \theta_{f}}{e_{B}}
$$

$$
\begin{aligned}
\bar{G}= & \frac{V w \sqrt{1-\sin \theta \cos \alpha}}{\left(B_{M}+1\right)} \\
& {\left[\left(B_{M}-1\right)-\frac{w^{2} e^{B}\left(1-\sin ^{2} \theta \cos ^{2} \alpha\right)}{\theta_{f} C_{A}}+\frac{B_{O}}{\theta_{f} C_{A}}\right] }
\end{aligned}
$$

\section{REFERENCES}

1. Bakr, A. A., Gelhar, L. W., Gutjahr, A. L. \& MacMillan, J. R. Stochastic analysis of spatial variability in subsurface flows 1. Comparison of one- and three dimension flows. Water Resour. Res., 1978, 14, 263-270.

2. Bentsen, R. G. A new approach to instability theory in porous media. Society of Petroleum Engineers, 1985, 25, 765-779.

3. Cary, J. W., Mcbride, J. F. \& Simmons, C. S. Observation of water and oil infiltration into soil: some simulation challenges. Water Resour. Res., 1989, 25, 73-80.

4. Chang, W.-L. T., Fractal analysis of wetting front instability in unsaturated soil. Ph.D. thesis, Dep. of Soil Sci., University of California, Davis, 1989.

5. Chuoke, R. L., Van Meurs, P. \& Van Der Pol, C. The instability of slow, immiscible, viscous liquid displacement in Permeable media petrol. Trans. AIME, 1959, 216, 188194.

6. Dagan, G. Solute transport in heterogeneous porous formation. J. Fluid Mech., 1984, 145, 151-177.

7. Davis, P. J. and Rabinowitz, P., Methods of Numerical Integration, 2nd edn, Academic, San Diego, CA, 1984.

8. Fetter, C. W., Contaminant Hydrogeology. Macmillan Inc., New York, 1993.

9. Freeze, R. A. A stochastic-conceptual analysis of 
one-dimension groundwater flow in nonuniform homogeneous media. Water Resour. Res., 1975, 11, 725-740.

10. Gelahr, L. W. \& Axness, C. L. Three-dimensional stochastic analysis of macro dispersion in aquifer. Water Resour. Res., 1983, 19, 161-180.

11. Glass, R. J. Immiscible displacement in porous media: stability analysis of three-dimension, axisymmetric disturbances with application to gravity-driven wetting front instability. Water Resour. Res., 1991, 27, 1947-1956.

12. Glass, R. J., Parlange, J.-Y. \& Steenhuis, T. S. Wetting front instability 1 . Theoretical discussion and dimension analysis. Water Resour. Res., 1989, 25, 1187-1194.

13. Green, W. H. \& Ampt, G. A. Studies in soil physics 1. The flow of water and air through soils. J. Agric. Soc., 1911, 4, 124.

14. Hill, S. Channelling in packed columns. Chem. Eng. Sci., 1952, 1, 247-253.

15. Hill, D. E. \& Parlange, J.-Y. Wetting front instability in layered soils. Soil Sci. Am. Proc., 1972, 36, 697-702.

16. Hunt, J. R., Sitar, N. \& Udell, K. S. Nonaqueous phase liquid and cleanup 1. Analysis of mechanisms. Water Resour. Res., 1988, 24, 1247-1258.

17. Kiefer, E. M. A conceptual-stochastic model of unsaturated flow in heterogeneous soils. J. of Hydrology, 1993, 143, 3-9.

18. Kueper, B. H. \& Frind, E. O. An overview of immiscible fingering in porous media. J. Contam. Hydrol., 1988, 2, 95 110 .

19. Larson, R. G., Scriven, L. E. \& Davis, H. T. Percolation theory of two phase flow in porous media. Chemical Engineering Science, 1981, 36, 57-73.

20. Li, S. G. \& McLaughlin, D. A nonstationary spectral method for solving stochastic groundwater problem: unconditional analysis. Water Resour. Res., 1991, 27, 1589-1605.

21. Lumley, J. L. and Panofsky, H. A., The structure of atmospheric turbulence. Wiley Interscience, New York, 1964.

22. Mantoglou, A. \& Gelhar, L. W. Stochastic modeling of largescale transient unsaturated flow systems. Water Resour. Res. $1987,23,37-46$.

23. Mantoglou, A. \& Gelhar. L. W. Capillary tension head variancc, mean soil moisture content, and effective specific soil moisture capacity of transient unsaturated flow in stratified solis. Water Resour. Res., 1987, 23, 47-56.

24. Mantoglou, A. \& Gelhar, L. W. Effective hydraulic conductivities of transient unsaturated flow in stratified soils. Water Resour. Res., 1987, 23, 57-67.

25. Mclean, J. W. \& Saffman, P. G. The effect of surface tension on the shape of fingers in a Hele-Shaw cell. J. Fluid Mech., $1981,102,455-469$.

26. Parlange, J.-Y. \& Hill, D. E. Theoretical analysis of wetting front instability in soils. Soil Sci., 1976, 122, 236-239.

27. Philip, J. R. Stability analysis of infiltration. Soil. Sci. Soc. Am. Proc., 1975, 39, 1042-1049.

28. Philip, J. R. The growth of disturbance in unstable infiltration flows. Soil. Sci. Soc. Am. Proc., 1975, 39, 1049-1053.

29. Raats, P. A. C. Unstable wetting fronts in uniform and nonuniform soils. Soil. Sci. Am. Proc., 1973, 37, 681-685.

30. Saffman, P. G. \& Taylor, G. I. The penetration of a fluid into a porous medium or Hele-Shaw cell containing a more viscous liquid. Proc. R. Soc. London. Ser. A., 1958, 246, 312 331.

31. Smith, L. \& Freeze, R. A. Stochastic analysis of steady state groundwater flow in a bounded domain 2. Two-dimension simulation. Water Resour. Res., 1979, 15, 1543-1559.

32. Unlu, K., Kavvas, M. L. \& Nielsen, D. R. Stochastic analysis of field measured unsaturated hydraulic Conductivity. Water Resour. Res., 1989, 25, 2511-2521.

33. Unlu, K., Nielsen, D. R. \& Biggar, J. W. Stochastic analysis of unsaturated flow: one-dimensional monte carlo simulations and comparisons with spectral perturbation analysis and field observations. Water Resour. Res., 1990, 26, 2207-2217.

34. Wei, W.-S. W., Time series analysis. Addison-Wesley, Redwood City, 1990.

35. Yeh, T.-C. J., Gelhar, L. W. \& Gutjahr, A. L. Stochastic analysis of unsaturated flow in heterogeneous soils 1 . Statistically isotropic media. Water Resour. Res., 1985, 21, 447456 